\title{
Interaction of viruses with host immune system and immunomodulation in chronic viral infections
}

\author{
Mengji Lu ${ }^{1 \bowtie}$, Yumei Wen ${ }^{2 \bowtie}$
}

1. Institute for Virology, University Hospital of Essen, University Duisburg-Essen, 45122 Essen, Germany; 2. Key Laboratory of Medical Molecular Virology of Ministries of Education and Health, Shanghai Medical College and Institute of Medical Microbiology, Fudan University, Shanghai 200032, China

The WHO estimates that almost 500 million people world-wide are chronically infected with hepatitis B and $\mathrm{C}$ viruses (HBV and $\mathrm{HCV}$ ) and human immune deficiency virus (HIV). During the recent years, significant progress was made in treatment of chronic infection with HBV, $\mathrm{HCV}$, and HIV, mainly based on directly antiviral agents. However, major challenges for the medical research remain. The current treatment regimens for HBV and HIV do still not sufficiently achieve complete viral eradication in patients. The development of prophylactic and therapeutic vaccines against HIV and HCV had suffered severe drawbacks. On the other hand, rapid advance in basic and infection immunology provided new insight into the interaction of host immune system and viruses, allowing the development of new concepts to stimulate innate and adaptive immunity to regain the control over viral infection. Though persistent infections with HBV, HCV, and HIV have distinct features and characteristics and the underlying mechanisms of viral persistence differ from each other, they are commonly associated with dysfunction of host immune system. This special issue is focusing on the interaction of the host immune system with HBV, HCV, and HIV and presents the latest advancement on those issues mentioned above.
The first part deals with the interaction of viruses with host immune cells and innate immune system. While the role of adaptive immunity in HBV infection has been intensely studied for many years, the contribution of innate immunity for the control of HBV infection is now recognized but still not fully understood. Chen et al., reviewed the interplay between HBV and the innate immune responses and discussed the implications for new therapeutic strategies (Chen J L, et al., 2014). Tucci and Küppers gave a perspective on the role of $\mathrm{HCV}$ in $\mathrm{B}$ cell lymphoproliferations (Tucci F, et al., 2014), an important aspect of HCV infection and pathogenesis. Ficolin is a kind of human serum complement lectin and acts as pattern recognition receptors in the host innate immunity. Its dysfunction or abnormal expression may play crucial roles in the viral, bacterial diseases, and inflammation. This review of Ren et al. summarized the current knowledge about ficolin functions in the infectious diseases and discussed the potential of ficolins as novel immunotherapeutics to treat these diseases (Ren Y S, et al., 2014).

Adaptive immune responses to viral infection, immune modulation, and viral escape are the focus of the second part of the special issue. Regulatory $\mathrm{T}$ cells (Tregs) are able to dampen effector $\mathrm{T}$ cell responses to viral infections and thereby contribute to the establishment of a chronic infection. The expansion, accumulation, and activation of Tregs in viral infections are of major interest in order to find ways to alter Treg functions for therapeutic benefit. In the Friend retrovirus (FV) mouse model, Tregs are has been extensively studied. Joedicke et al. analyzed the phenotype and activation status of regulatory $T$ cells during Friend retrovirus infection in detail (Joedicke J J, et al., 2014). Therapeutic vaccination becomes a promising strategy to induce potent and durable antiviral immune responses to HBV in chronically infected patients and to achieve long-term control of viral replication. Current published preclinical studies indicate the effectiveness of therapeutic vaccination. Liu et al., reviewed new therapeutic vaccination strategies to treat chronic hepatitis B which may be introduced for patient treatment in the future (Liu J, et al., 2014). HIV-1 evolves strongly and undergoes geographic differentiation as it spreads in diverse host populations around the world. Dang et al., asked whether this differentiation affects the selection pressure experienced by the virus and evaluated selection pressure on the HIV-1 envelope protein gp120 of subtype B and east Asian variant $\mathrm{B}^{\prime}$ at the level of individual codon using different 
bioinformatic methods (Dang $\mathrm{S}$, et al., 2014). HCV has the capacity to evolve rapidly and thereby evade host immune pressure. The genetic heterogeneity is an important feature of HCV. Different HCV stains may differ strongly in their biological and functional characteristics. Cao et al., reported a new strain of chimeric hepatits $\mathrm{C}$ virus isolated from a chronic hepatitis $\mathrm{C}$ patient from China which could be used a reference to compare with stains from other origins (Cao H, et al., 2014).

The final part of the special is dedicated to vaccine development against viral infections. Temchura and Tenbusch reviewed the recent concepts and approaches of HIV vaccine development and discussed the complex consequences of vaccine-induced immune response, protection against $\mathrm{HIV}$ at one site and increased risk of HIV infection by activation of CD4 T-cells on the other site (Temchura V, et al., 2014). Recently, structured vaccines based on nanoparticles gain great attention, as they could facilitate the uptake of antigens by antigen-presenting cells. In the present work of Kozlova et al., generated biodegradable calcium phosphate nanoparticles were combined with flagellin, known as TLR5 ligand (Kozlova D, et al., 2014). Such nanoparticles showed an effective activation of the innate immune response in vitro and in vivo, indicating their potential as vaccine carriers.

When you closely read the papers in this issue, you may find those researches are commonly founded by a so-called TRR60 program. The Transregional Collaboration Research Project (TRR) is a highly ranked program of German Research Foundation (DFG) to support the scientific competence with a strong focus on a specific research area. The joint German-Chinese TRR60 was firstly established by DFG in collaboration with the Ministry of Science and Technology (MOST) of China in 2009 and renewed by The National Science Foundation of China (NSFC) and DFG in 2013. The TRR60 recruited recognized scientists in the research field on viral infection and immunology from both countries. This research program is a result of the great effort of Chinese and German scientists, represents a milestone in the history of long standing and fruitful collaboration, and serves as a platform for active interaction and exchange.

We would like to thank the TRR60 program, all authors for their excellent contributions to this special issue, and the editorial office of Virologica Sinica for their constant support.

\section{FOOTNOTES}

This work was supported by the DFG through SFB TRR60.

Published ahead of print:

22 January 2014

Corresponding authors.

Mengji Lu:

Email: mengji.lu@uni-due.de

Yumei Wen:

Email:ymwen@shmu.edu.cn

\section{REFERENCES}

Cao H, Zhu W, Han Q X, et al. 2014. Virol Sin, 29: 61-70

Chen J L and Yuan Z H. 2014. Virol Sin, 29: 17-24.

Dang S, Wang Y, Budeus B, et al. 2014. Virol Sin, 29: 40-47.

Joedicke J J, Dietze K K, Zelinskyy G, et al. 2014. Virol Sin, 29: 48-60.

Kozlova D, Sokolova V, Zhong M H, et al. 2014. Virol Sin, 29: 33-39.

Liu J, Kosinska A, Lu M J, et al. 2014. Virol Sin, 29: 10-16.

Ren Y S, Ding Q Q and Zhang X L. 2014. Virol Sin, 29: 25-32.

Temchura V and Tenbusch M. 2014. Virol Sin, 29: 7-9.

Tucci F and Küppers R. 2014. Virol Sin, 29: 3-6. 